\title{
A Study on Association between Neutrophil to Lymphocyte Ratio and Steatohepatitis and Fibrosis in Patients with Non-Alcoholic Fatty Liver Disease
}

\author{
Jayachandra ${ }^{1}$, Sree Raksha K.N², Rakshit R. Desai ${ }^{3}$, Chetan V4, Arjun P. Chandrashekar ${ }^{5}$
}

1,2,3,4,5 Department of General Medicine, Bangalore Medical College and Research Institute, Bangalore, Karnataka, India.

\section{ABSTRACT}

\section{BACKGROUND}

Non-alcoholic fatty liver disease (NAFLD) is seen worldwide and is the most common liver disorder in industrialized countries. Non-alcoholic steatohepatitis (NASH) is the severest form of NAFLD and is associated with inflammation and oxidative stress. Human neutrophil peptides have the ability to enhance hepatic fibrosis. We wanted to study the association between neutrophil to lymphocyte ratio and steatohepatitis and fibrosis in patients with non-alcoholic fatty liver disease.

\section{METHODS}

This is an analytical cross-sectional study conducted among 50 cases and 50 controls attending the hospital attached to BMCRI. The neutrophil lymphocyte ratio was calculated and compared between cases and controls.

\section{RESULTS}

Our study results showed that 7 patients had grade 1, 19 had grade 2, 24 had grade 3 hepatic steatosis and 13 patients had fibrosis. The mean neutrophil lymphocyte ratio (NLR) was significantly higher in cases than controls (3.6 +/- 1.83 and $1.72+/-0.57$, $P$ - 0.00). However, there was no statistical significance (F: 2.06 , $P$ value: 0.14 ) in the utility of NLR in detecting higher grades of steatosis (grade 1:2.41+/- 0.76 , grade 2:4.01 +/- 2.46, grade 3:3.62 +/- 1.3). The mean NLR was significantly higher ( $\mathrm{P}$ $0.001)$ in patients with fibrosis $(3.72+/-2.1)$ compared with controls $(1.46+/-051)$.

\section{CONCLUSIONS}

The neutrophil lymphocyte ratio can be used to detect the presence of steatohepatitis and fibrosis in patients with NAFLD but cannot be used to predict the presence of higher grades of hepatic steatosis.

\section{KEY WORDS}

Non-Alcoholic Fatty Liver Disease, Non-Alcoholic Steatohepatitis, Fibrosis, Neutrophil-Lymphocyte Ratio
Corresponding Author: Dr. Rakshit R. Desai, Post Graduate Student, Department of General Medicine, Bangalore Medical College and Research Institute, Bangalore, Karnataka, India. E-mail: rd57180@gmail.com

DOI: $10.14260 / j e m d s / 2021 / 388$

How to Cite This Article:

Jayachandra, Raksha KNS, Desai RR, et al. A study on association between neutrophil to lymphocyte ratio and steatohepatitis and fibrosis in patients with non-alcoholic fatty liver disease. J Evolution Med Dent Sci 2021;10(25):1876-1882, $10.14260 /$ jemds/2021/388 DOI:

Submission 12-08-2020,

Peer Review 02-04-2021,

Acceptance 08-04-2021,

Published 21-06-2021.

Copyright $(2021$ Jayachandra et al. This is an open access article distributed under Creative Commons Attribution License [Attribution 4.0 International (CC BY 4.0)] 


\section{BACKGROUND}

Non-alcoholic fatty liver disease is one of the most common causes of chronic liver disease. ${ }^{1}$ Non-alcoholic fatty liver disease encompasses a spectrum of pathologic conditions, ranging from simple fatty infiltration termed as steatosis to non-alcoholic steatohepatitis (NASH) and cirrhosis. ${ }^{2}$ This entity has now reached epidemic proportions and is now one of the leading indication for liver transplantation in Western countries. ${ }^{3}$ Though the pathological picture resembles that of alcohol induced liver injury, it occurs in patients who do not consume alcohol or consume alcohol in quantities not considered to be harmful to the liver (less than $20 \mathrm{~g} /$ day or < $140 \mathrm{~g} /$ week). 4

NAFLD is characterized by deposition of triglyceride in the liver greater than $5 \%$ of the total liver weight without a history of excessive alcohol intake or known aetiologies of liver disease. ${ }^{4,5}$ Approximately 20 to $30 \%$ of adults in the general population in Western countries have NAFLD, and its prevalence increases to 70 to $90 \%$ among persons who are obese or have diabetes. ${ }^{6}$ The overall prevalence of NAFLD is 15 to $40 \%$ in western countries while $9-40 \%$ in Asian countries. $^{7}$

Diabetes mellitus (DM), obesity and hyperinsulinemia are associated with NAFLD. ${ }^{8}$ There has been an exponential increase in the incidence of DM, obesity, and insulin resistance in India in the last two decades. ${ }^{9,10}$ Prevalence of NAFLD based on the ultrasound is $18.9 \%$ in adults according to a population-based study in western India. ${ }^{7}$

Non-alcoholic steatohepatitis is the most severe form of non-alcoholic fatty liver disease and is associated with inflammation and oxidative stress. Although, dysregulated lipid accumulation occurs across the non-alcoholic fatty liver disease spectrum, the features of liver cell injury, such as hepatocyte ballooning, cytoskeletal changes and hepatocyte apoptosis occur predominantly in NASH and distinguish NASH from simple steatosis. ${ }^{11}$

Simple steatosis without fibrosis or inflammation has a benign clinical course. NASH, however, has a more progressive course and can lead to cirrhosis in $10-15 \%$ of the patients. A liver biopsy remains the gold standard to establish extent of liver damage and fibrosis and is an invasive and expensive procedure. Imaging studies have been used to diagnose NAFLD with less sensitivity and specificity. Hence there is a great need for identifying non-invasive markers for predicting NASH and significant fibrosis. Blood neutrophil-lymphocyte ratio is a simple marker of subclinical inflammation and can be easily calculated from the differential WBC counts. The N/L ratio has been used to predict outcomes in patients with coronary artery disease and cancer.

Neutrophil-lymphocyte ratio integrates information on the inflammatory milieu and physiological stress. A prominent feature of inflammation is neutrophil accumulation. Neutrophil derived myeloperoxidase can increase macrophage cytotoxicity and induce neutrophil activation in a NASH mouse model.12

In the foz/foz NASH metabolic syndrome model, a reduction in the hepatic cholesterol stores was associated in ameliorated liver injury, apoptosis, macrophage, and neutrophil accumulation. Neutrophil dysfunction was also associated with liver fibrosis and cirrhosis in NASH. ${ }^{13}$ Human neutrophil peptides have the ability to enhance liver fibrosis in fatty liver disease by inducing hepatic stellate cell proliferation. ${ }^{14}$

This ratio integrates information on two different pathways - the neutrophil that is responsible for ongoing inflammation and the lymphocytes that represent the regulatory pathway. The N/L ratio is an indicator of overall inflammatory status of the body and higher ratios may be found in patients with NAFLD patients with advanced disease. ${ }^{14}$ The neutrophil-lymphocyte ratio can hence be used as a marker for NASH and fibrosis in patients with nonalcoholic fatty liver disease and can be a novel, non-invasive marker to predict advanced disease.

We wanted to study the association between neutrophil to lymphocyte ratio and steatohepatitis and fibrosis in patients with non-alcoholic fatty liver disease.

\section{METHODS}

This is an analytical cross-sectional study conducted from November 2016 to October 2018. Data was collected from the patients visiting the outpatient department and in-patients at hospitals attached to Bangalore Medical College and Research Institute.

\section{Sample Size}

Based on a previous study by Naim Alkhouri et al. neutrophil value was $4.1 \pm 1.4$ for cases and assumed equal standard deviation for controls and minimum expected difference is 0.8 .15

$n=\frac{2\left(Z_{\alpha}+Z_{1-\beta}\right)^{2} \sigma^{2}}{d^{2}}$

$n=\frac{2(1.96+0.84)^{2}(1.4)^{2}}{(0.8)^{2}}$

$n=48 \simeq 50$

50 cases and 50 age and sex matched healthy controls were considered for the study.

\section{Inclusion Criteria}

1. Patients willing for study

2. Age $\geq 18$ years,

3. Non-alcoholics

4. Raised liver enzymes - AST, ALT at least 1.5 times above upper limit of normal

5. USG examination showing - Diffuse fatty infiltration

\section{Diffuse Fatty Infiltration will Be Graded as Follows -}

1. Mild/grade 1 (mild steatosis) - slightly increased liver echogenicity with normal vessels and absent posterior attenuation

2. Moderate/grade 2 (moderate steatosis) - moderately increased liver echogenicity with partial dimming of vessels and early posterior attenuation 
3. Severe /grade 3 (severe steatosis) - diffusely increased liver echogenicity with absence of visible vessels and heavy posterior attenuation.

\section{Exclusion Criteria}

1. Positive test for HBsAg and anti HCV antibodies

2. Alcohol intake $>20 \mathrm{gm} /$ day

3. Past history of chronic liver disease of proven aetiology.

4. Patients on medications that can induce fatty liver like methotrexate, oestrogen, amiodarone, tamoxifen, corticosteroids and tetracyclines

5. Hepatocellular carcinoma

6. History of gastrointestinal bypass surgery

7. Patients with active infection.

8. Patients not consenting for the study.

Written informed consent was taken from the patients. After taking informed consent from patients, a detailed history and clinical examination was performed to identify all the necessary symptoms and signs.

All the routine investigations such as

- Complete blood counts

- Liver function tests

- Renal function tests

- Blood sugars

- Viral markers (HBsAg, anti - HCV antibodies)

- Ultrasound abdomen

- Other relevant investigations

Non-alcoholic fatty liver disease was graded as per ultrasonographic criteria.

USG examination showing - diffuse fatty infiltration. Diffuse fatty infiltration will be graded as follows: 16

- Mild/grade 1 (mild steatosis) - slightly increased liver echogenicity with normal vessels and absent posterior attenuation

- Moderate/grade 2 (moderate steatosis) - moderately increased liver echogenicity with partial dimming of vessels and early posterior attenuation

- Severe/grade 3 (severe steatosis) - diffusely increased liver echogenicity with absence of visible vessels and heavy posterior attenuation.

- Fibrosis was evidenced as increase in the echotexture of the liver with surface nodularity.

Neutrophil-lymphocyte ratio is calculated from the differential count as reported in $\mathrm{CBC}$ and was compared with that of controls.

\section{Statistical Analysis}

The data was entered in Microsoft excel sheet and was analysed using statistical package for social sciences (SPSS) version 22 software. The categorical data was represented in the form of frequency and percentage. Chi-square/Fisher's exact test was used to test the significance for categorical data.

Continuous data was represented as mean and standard deviation. Student - $t$ test and analysis of variance (ANOVA) was used for comparison between groups. $\mathrm{P}$ value $<0.05$ was considered statistically significant.

\section{RESULTS}

Table 1 shows distribution of the subjects based on age. Equal distribution was considered in both cases and controls with higher distribution of subjects in 41 to 60 years age group - (20 out of 100). Equal distribution was considered in both cases and controls with respect to gender with maximum subjects being females - 68 out of 100 .

\begin{tabular}{|c|c|c|c|c|}
\hline & Age & Case & Control & Total \\
\hline \multirow{6}{*}{$\begin{array}{l}\text { Distribution } \\
\text { of the } \\
\text { subjects } \\
\text { based on age }\end{array}$} & $\leq 20$ & $\begin{array}{c}2 \\
50.0 \%\end{array}$ & $\begin{array}{c}2 \\
50.0 \%\end{array}$ & $\begin{array}{c}4 \\
100.0 \%\end{array}$ \\
\hline & $21-40$ & $\begin{array}{c}17 \\
50.0 \%\end{array}$ & $\begin{array}{c}17 \\
50.0 \%\end{array}$ & $\begin{array}{c}34 \\
100.0 \%\end{array}$ \\
\hline & $41-60$ & $\begin{array}{c}20 \\
50.0 \%\end{array}$ & $\begin{array}{c}20 \\
50.0 \%\end{array}$ & $\begin{array}{c}40 \\
100.0 \%\end{array}$ \\
\hline & $61-80$ & $\begin{array}{c}10 \\
50.0 \%\end{array}$ & $\begin{array}{c}10 \\
50.0 \%\end{array}$ & $\begin{array}{c}20 \\
100.0 \%\end{array}$ \\
\hline & $>80$ & $\begin{array}{c}1 \\
50.0 \%\end{array}$ & $\begin{array}{c}1 \\
50.0 \%\end{array}$ & $\begin{array}{c}2 \\
100.0 \%\end{array}$ \\
\hline & Total & $\begin{array}{c}50 \\
50.0 \%\end{array}$ & $\begin{array}{c}50 \\
50.0 \%\end{array}$ & $\begin{array}{c}100 \\
100.0 \%\end{array}$ \\
\hline \multirow{4}{*}{$\begin{array}{l}\text { Distribution } \\
\text { of the } \\
\text { subjects } \\
\text { based on } \\
\text { gender }\end{array}$} & Gender & Case & Control & Total \\
\hline & Male & $\begin{array}{c}16 \\
50.0 \%\end{array}$ & $\begin{array}{c}16 \\
50.0 \%\end{array}$ & $\begin{array}{c}32 \\
100.0 \%\end{array}$ \\
\hline & Female & $\begin{array}{c}34 \\
50.0 \%\end{array}$ & $\begin{array}{c}34 \\
50.0 \%\end{array}$ & $\begin{array}{c}68 \\
100.0 \%\end{array}$ \\
\hline & Total & $\begin{array}{c}50 \\
50.0 \%\end{array}$ & $\begin{array}{c}50 \\
50.0 \%\end{array}$ & $\begin{array}{c}100 \\
100.0 \%\end{array}$ \\
\hline
\end{tabular}

\begin{tabular}{|ccc|}
\hline Symptoms & Cases \\
Malaise & 41 & Percent \\
Abdominal discomfort & 27 & 82.0 \\
Jaundice & 7 & 54.0 \\
Abdominal distension & 3 & 14.0 \\
Fever & 2 & 6.0 \\
\hline Table 2A. Distribution of the Subjects Based on Symptoms \\
\hline
\end{tabular}

\begin{tabular}{|ccc|}
\hline BMI (Asian Classification) & Cases & \\
$18.5-22.9$ & 5 & Percent \\
$23-24.9$ & 2 & 10.0 \\
$\geq 25$ & 43 & 4.0 \\
Total & $\mathbf{5 0}$ & 86.0 \\
\hline Table 2B. Distribution of the Subjects Based on BMI \\
\hline
\end{tabular}

Out of 50 cases, 23 (46\%) patients had diabetes and 8 (16 $\%)$ cases had hypertension. Out of $50(100 \%), 43(86 \%)$ had $\mathrm{BMI} \geq 25$ followed by 5 (10\%) had BMI in the range of 18.5 22.9 and the least number of subjects $-2(4 \%)$ were in the BMI range of 23 - 24.9. Cross-tabulation of gender and waist circumference showed that out of 34 (68 \%) females, 23 (95.8 $\%)$ were obese and 11 (42.3\%) were non-obese where - as out of 16 (32\%) males, 15 (57.7\%) were non-obese and 1 (4.2\%) was obese.

\begin{tabular}{|c|cc|}
\hline Signs & \multicolumn{2}{|c|}{ Cases } \\
Hepatomegaly & Frequency & Percent \\
Pedal oedema & 27 & 54.0 \\
Abdominal tenderness & 21 & 42.0 \\
Pallor & 12 & 24.0 \\
Icterus & 11 & 22.0 \\
\hline Table 3. Distribution of the Subjects Based on Signs \\
\hline
\end{tabular}

Other signs observed among cases showed that hepatomegaly was high among cases ( $54 \%$ ), followed by pedal oedema (42\%). The mean haemoglobin of cases and controls was $12.02 \pm 1.94$ and $12.9 \pm 1.35$ respectively.

The difference between mean haemoglobin and the groups was significant $(\mathrm{P}-0.000)$. The mean total count of cases and controls was $9,636.02+2,990.82$ and $7,920.80+2,276.91$ 
respectively. The difference between mean total count and the groups was significant $(\mathrm{P}-0.000)$

The mean neutrophils of cases and controls was $71.15 \pm$ 8.35 and $57.58 \pm 7.38$ respectively. The difference between neutrophils and the groups was significant $(P-0.000)$. The mean lymphocytes of cases and controls was $22.33 \pm 6.13$ and $35.27+6.48$ respectively. The difference between lymphocytes and the groups was significant $(P-0.000)$. The mean platelet of cases and controls was $2.31+0.85$ and $2.93+$ 0.99 respectively. The difference between mean platelet count and the groups was significant $(\mathrm{P}-0.000)$

The mean MCV of cases and controls was $84.85+8.41$ and $84.23 \pm 11.93$ respectively. The difference between mean MCV and the groups was not significant ( $\mathrm{P}-0.77)$. The mean RBS of cases and controls was $181.36 \pm 108.82$ and $111.66 \pm 112.97$ respectively. The difference between mean RBS and the groups was significant $(P-0.00)$. The mean urea of cases and controls was $27.62 \pm 10.21$ and $23.24 \pm 6.89$ respectively. The difference between mean urea and the groups was significant (P - 0.01). The mean creatinine of cases and controls was 0.78 \pm 0.34 and $0.69 \pm 0.20$ respectively. The difference between mean creatinine and the groups was not significant $(\mathrm{P}-0.11)$

The mean total bilirubin of cases and controls was $1.55 \pm$ 0.85 and $0.59 \pm 0.26$ respectively. The difference between mean total bilirubin and the groups was significant $(\mathrm{P}-0.00)$. The mean direct bilirubin of cases and controls was $0.65 \pm 0.46$ and $0.20 \pm 0.15$ respectively. The difference between mean direct bilirubin and the groups was significant $(\mathrm{P}-0.00)$. The mean total protein of cases and controls was $6.22 \pm 0.8$ and $7.12 \pm 0.6$ respectively. The difference between mean total protein and the groups was significant $(\mathrm{P}-0.00)$

The mean albumin of cases and controls was $2.98 \pm 0.77$ and $3.81 \pm 0.47$ respectively. The difference between mean albumin and the groups was significant $(\mathrm{P}-0.00)$. The mean globulin of cases and controls was $3.23 \pm 0.82$ and $3.27 \pm 0.54$ respectively. The difference between mean globulin and the groups was not significant $(\mathrm{P}-0.77)$

\begin{tabular}{|c|c|c|c|c|c|}
\hline Variables & Group & Mean & $\begin{array}{l}\text { Standard } \\
\text { Deviation }\end{array}$ & t Test & $\begin{array}{c}P \\
\text { Value }\end{array}$ \\
\hline AST & $\begin{array}{c}\text { Case } \\
\text { Control }\end{array}$ & $\begin{array}{l}86.66 \\
22.84\end{array}$ & $\begin{array}{c}39.70 \\
9.23\end{array}$ & 11.07217994 & 0.00 \\
\hline ALT & $\begin{array}{l}\text { Case } \\
\text { Control }\end{array}$ & $\begin{array}{l}91.50 \\
25.14\end{array}$ & $\begin{array}{l}25.34 \\
25.20\end{array}$ & 13.12868604 & 0.00 \\
\hline ALP & $\begin{array}{l}\text { Case } \\
\text { Control }\end{array}$ & $\begin{array}{c}157.46 \\
84.24\end{array}$ & $\begin{array}{l}45.61 \\
27.16\end{array}$ & 9.753292441 & 0.00 \\
\hline NLR & $\begin{array}{c}\text { Case } \\
\text { Control }\end{array}$ & $\begin{array}{l}3.60 \\
1.72\end{array}$ & $\begin{array}{l}1.83 \\
0.57\end{array}$ & 6.940614989 & 0.00 \\
\hline $\begin{array}{l}\text { Tab } \\
\text { Lyn }\end{array}$ & $\begin{array}{l}\text { Compo } \\
\text { ocyte Ro }\end{array}$ & $\begin{array}{l}\text { on of } \\
\text { (NLR }\end{array}$ & $\begin{array}{l}T, A L T, A L I \\
\text { etween } C a\end{array}$ & $\begin{array}{l}\text { nd Neutrophi } \\
\text { and Control }\end{array}$ & \\
\hline
\end{tabular}

The mean AST of cases and controls was $86.66 \pm 39.7$ and $22.84 \pm 9.23$ respectively. The difference between mean AST and the groups was significant (P - 0.00). The mean ALT of cases and controls was $91.50 \pm 25.34$ and $25.14 \pm 25.20$ respectively. The difference between mean ALT and the groups was significant $(\mathrm{P}-0.00)$. The mean ALP of cases and controls was $157.46 \pm 45.61$ and $84.24 \pm 27.16$ respectively. The difference between mean ALP and the groups was significant $(P-0.00)$. The mean NLR of cases and controls was $3.6 \pm 1.83$ and $1.72 \pm 0.57$ respectively. The difference between mean NLR and the groups was significant $(P-0.00)$.

Mean distribution of grading of HS and NLR showed higher mean with grade $2(4.01 \pm 2.46)$ followed by grade $3(3.62 \pm$ $1.3)$ and grade $1(2.41 \pm 0.76)$. ANOVA was applied to find the statistical difference among the grading of HS. ANOVA showed no statistically significant difference among the groups for mean NLR $(\mathrm{F}=2.06 ; \mathrm{P}=0.14)$.

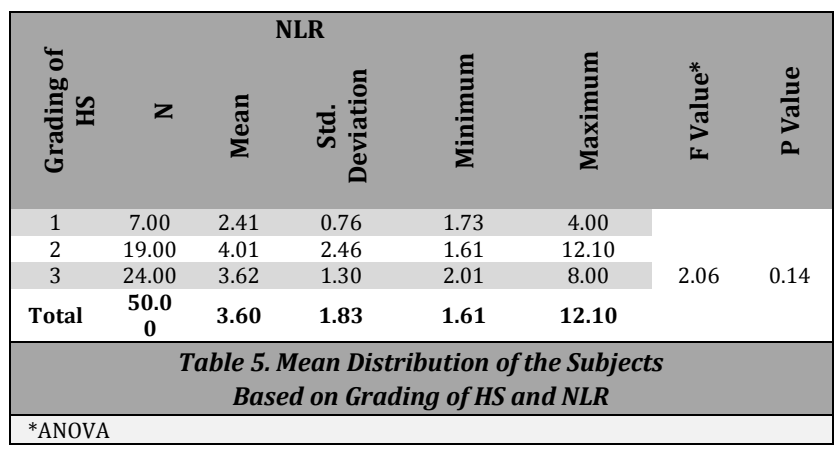

\begin{tabular}{|cccccccc|}
\hline Fibrosis & N & Mean & Std. Dev. & $\begin{array}{c}\text { Mini- } \\
\text { mum }\end{array}$ & $\begin{array}{c}\text { Maxi- } \\
\text { mum }\end{array}$ & $\begin{array}{c}\text { t } \\
\text { Test }\end{array}$ & $\begin{array}{c}\text { P } \\
\text { Value }\end{array}$ \\
Yes & 13 & 3.27 & 0.50 & 2.1 & 4.50 & 9.06 & 0.001 \\
No & 13 & 1.46 & 0.51 & 0.97 & 2 & 5 & 0.07 \\
\hline \multicolumn{7}{c|}{ Table 6. Mean Distribution of the Subjects } \\
Based on Fibrosis and NLR
\end{tabular}

Mean distribution of fibrosis and NLR showed higher mean with fibrosis $(3.72 \pm 2.1)$ as compared to mean NLR without fibrosis $(1.46 \pm 0.51)$. Independent sample t test was applied to find the statistical difference between the fibrosis. There was statistically significant difference between the groups for mean NLR ( $\mathrm{T}=9.065 ; \mathrm{P}=0.001)$.

\section{DISCUSSION}

Chronic liver disease is one of the leading causes of significant morbidity and mortality accounting to 800,000 deaths per year. ${ }^{17}$ Non-alcoholic fatty liver disease is one of the most common causes of liver disease in modern industrialized economies. NAFLD is highly prevalent in modern societies and 10 - $25 \%$ cases develop hepatic fibrosis leading to cirrhosis, end stage liver disease and hepatocellular carcinoma. ${ }^{18}$

Liver biopsy is the "gold standard test" for diagnosis and staging of the disease. It is invasive and cumbersome. Studies on non-invasive methods in identifying severe disease is an area of active research and the search for a novel non-invasive marker continues.

Neutrophil lymphocyte ratio could be an important marker of systemic inflammation. The increase in NLR could be explained by the "Two hit hypothesis." The first hit is excessive triglyceride accumulation, and the second hit is activated pro-inflammatory pathways. NLR has been related to various inflammatory conditions and cancers and has been associated with poorer outcomes. ${ }^{19-24}$

In our study conducted at Bangalore medical college and research institute and attached hospitals, 50 cases of NAFLD and 50 age and sex matched controls were compared. It was found that 37 (74\%) patients belonged to the age group between 20 - 60 years of age proving that the disease prevailed in the young and middle aged with maximum patients in the age group between 40 - 60years. $32 \%$ cases were males and $68 \%$ patients were females. In a population-based study by Deepak Amarapurkar et al. ${ }^{7}$ it was found that the mean age of the patients with NAFLD was $39.08+/-12.3$ years and the disease was prevalent amongst the middle aged. However, the 
prevalence of NAFLD was higher in males compared to females.

41(82\%) patients complained of "malaise" which was the predominant symptom followed by abdominal discomfort in 27 (54\%) patients and loss of appetite in $9(18 \%)$ patients, proving that patients presented more commonly with nonspecific symptoms. Hepatomegaly was observed in 27 (54 \%) patients followed by pedal oedema in 21 (42\%) patients. 11 (22\%) patients had jaundice. According to a review article by Basaranoglu and Neuschwander -Tetri, 25 the most frequent symptoms are fatigue and right upper quadrant pain or dullness, although most patients may be asymptomatic, which is similar to the observations in our study. Mild to moderate hepatomegaly is one of the most common physical examination findings.

Non-alcoholic fatty liver disease has been increasing in prevalence in the last decade and has been found to have a strong association with metabolic risk factors of diabetes, dyslipidaemia and obesity. NAFLD is seen in more than $70 \%$ of patients with diabetes and coexistent obesity.

In a study by Deepak Amarapurkar et al. ${ }^{7}$ a populationbased study, diabetes was existent in $22 \%$ cases of NAFLD, 52 $\%$ cases had obesity and $38 \%$ patients had abdominal obesity. ${ }^{7}$ In a study by Singh SP et al.26 464 consecutive patients with NAFLD were studied for associated risk factors, diabetes was seen in $15.2 \%$ of cases, hypertension in $17.4 \%$ of the cases. The mean BMI observed in cases was $26.25+/$ 3.80 which is in accordance with our study.

In our study, 23 (46\%) patients had diabetes and 8 (16\%) cases had hypertension as existing comorbidities. $86 \%$ patients had body mass index (BMI) greater than $25 \mathrm{~kg} / \mathrm{m}^{2}$ and 4 patients were overweight and mean BMI was $27.652 \mathrm{~kg} / \mathrm{m}^{2}$, indicating that obesity is a major risk factor for the development of NAFLD. 24 (48\%) patients had higher waist circumference with majority of patients being females with abdominal obesity. A study by Ramesh Kumar et al. ${ }^{27} 205$ cases of NAFLD were studied, it was observed that $68.8 \%$ patients were obese and $18 \%$ were overweight.

White blood cell (WBC) count is a simple, global and inexpensive marker of inflammation and is a useful marker for infection and inflammation. Higher levels of WBC count have been associated with diabetes and metabolic syndrome linked to chronic low-grade inflammation and insulin resistance. Insulin resistance is a key feature in the pathogenesis of NAFLD.

In our study, significant statistical difference $(\mathrm{P}<0.05)$ was noted between cases and controls with respect to total counts, neutrophil counts, lymphocyte counts and platelet count. Mean total count was 9,636 $\pm 2,990.82$ in cases and 7,920.80 \pm $2,276.91$ respectively. The total counts were significantly higher in cases than controls. In a cross-sectional study by Yong - Jae Lee et al. ${ }^{28}$ a positive association was found between WBC counts and NAFLD prevalence in a dose response manner. In a study by Kuppan et al. ${ }^{29}$ it was found that the leucocyte count was significantly higher in patients with NAFLD compared with those without $\left(7.8 \pm 1.4 \times 10^{3}\right.$ vs. $6.9 \pm$ $0.9 \times 10^{3}$, P value < 0.001) .

The mean neutrophil percentage was $71.15 \pm 8.35 \%$ and $57.58 \pm 7.38 \%$ in cases and controls respectively and was found to be higher in cases compared to controls and was statistically significant. The mean lymphocyte percentage in cases and controls were $22.33 \pm 6.13 \%$ and $35.27 \pm 6.48 \%$ respectively and it was statistically significant. Lower lymphocyte counts were found in cases compared to controls. The mean platelet count in cases and controls were $2.31 \pm 0.85$ $\mathrm{lakh} / \mathrm{mm}^{3}$ and $2.93 \pm 0.99 \mathrm{lakh} / \mathrm{mm}^{3}$ respectively and was found to be statistically significant in our study.

The cases had a lower mean platelet count compared to the controls. The peripheral platelet production is regulated by thrombopoietin which is synthesized in the liver. In a prospective cohort study by Liu et al. ${ }^{30}$ at five years of follow up, there was significant reduction in the platelet count from $220 \pm 40.70\left(10^{9} / \mathrm{L}\right)$ to $208.41 \pm 43.26\left(10^{9} / \mathrm{L}\right)$ in patients with NAFLD even after adjustment for confounding variables.

The mean total bilirubin was $1.55 \pm 0.85 \mathrm{mg} / \mathrm{dl}$ and $0.59 \pm$ $0.26 \mathrm{mg} / \mathrm{dl}$ in cases and controls respectively. The mean direct bilirubin of cases and controls was $0.65 \pm 0.46 \mathrm{mg} / \mathrm{dl}$ and 0.20 $\pm 0.15 \mathrm{mg} / \mathrm{dl}$ respectively. The mean total bilirubin and direct bilirubin was significantly higher in cases and controls.

In a study by Chang Y et al. ${ }^{31}$ it was found that higher direct bilirubin levels were significant associated with a lower risk of developing NAFLD. On the contrary our study shows significantly higher levels of total and direct bilirubin levels in cases compared to controls.

Mean albumin level was $2.98 \pm 0.77 \mathrm{~g} / \mathrm{dl}$ in cases and 3.81 $\pm 0.47 \mathrm{~g} / \mathrm{dl}$ in controls and albumin levels were significantly lower in cases. However, there was no significant statistical difference for globulin levels. Younossi et al. ${ }^{32}$ proved that hypoalbuminemia, high total bilirubin, and prolonged prothrombin time in patients with diabetes mellitus and NAFLD was associated with poorer outcome.

Cases had significantly higher mean AST, ALT and ALP levels compared to controls and ALT levels were higher than AST levels. In a study by Chang et al. ${ }^{33}$ it was observed that in apparently health non-diabetic Korean men, increasing ALT concentration, even within its reference interval, was an independent predictor of incident NAFLD.

In our study, it was observed that 7 patients had grade 1 hepatic steatosis, 19 patients had grade 2 hepatic steatosis and 24 patients had grade 3 hepatic steatosis with 13 patients having evidence of fibrosis on ultrasonography. The mean neutrophil - lymphocyte ratio of cases and controls was $3.6 \pm$ 1.83 and $1.72 \pm 0.57$ respectively. The difference between mean NLR and the groups was significant (P - 0.00). Mean distribution of grading of HS and NLR showed higher mean with grade $2(4.01 \pm 2.46)$ followed by grade $3(3.62 \pm 1.3)$ and grade $1(2.41 \pm 0.76)$.

ANOVA showed no statistically significant difference among the groups for mean NLR ( $\mathrm{F}=2.06$; $\mathrm{P}=0.14)$. Mean distribution of fibrosis and NLR showed higher mean with fibrosis $(3.72 \pm 2.1)$ as compared to mean NLR without fibrosis $(1.46 \pm 0.51)$. There was statistically significant difference between the groups for mean NLR ( $\mathrm{T}=9.065 ; \mathrm{P}=0.001)$.

Observations in our study shows that the mean NLR was higher in cases compared to controls and it was statistically significant. Hence, NLR can be used to detect the presence of steatohepatitis in patients with NAFLD.

However, there was no statistical significance in the utility of NLR in detecting higher grades of hepatic steatosis. The mean NLR was significantly higher in patients with fibrosis compared with controls and hence can be used as a marker to identify fibrosis, which is advanced disease in patients with NAFLD. 
In a study conducted by Naim Alkhouri et al. ${ }^{34}$ it was found that the patients with NASH had higher NLR compared to the non-NASH group [2.5 (1.9 - 3.3) and $1.6(1.2$ - 2.0)] with P value $<0.001$ and patients with advanced fibrosis had elevated NLR [2.9 (2.0 - 3.9)] which was statistically significant which is similar to the observation in our study.

In a study by N.K. Kahraman et al. ${ }^{35}$ where the NLR was used to assess the severity of NAFLD in diabetics, it was found that neutrophil-lymphocyte ratio increases with increasing grade of steatosis in NAFLD patients with diabetes and can be used as a convenient marker to follow progression of nonalcoholic fatty liver disease. Our study failed to correlate with the increasing grade of steatosis but showed higher mean NLR in cases compared to controls and can be used as a noninvasive marker to detect the presence of NAFLD and to detect advanced disease.

\section{CONCLUSIONS}

Neutrophil lymphocyte ratio can be used to detect the presence of steatohepatitis in patients with NAFLD. The neutrophil lymphocyte ratio cannot be used to predict the presence of higher grades of hepatic steatosis.

Data sharing statement provided by the authors is available with the full text of this article at jemds.com.

Financial or other competing interests: None.

Disclosure forms provided by the authors are available with the full text of this article at jemds.com.

\section{REFERENCES}

[1] Dietrich P, Hellerbrand C. Non-alcoholic fatty liver disease, obesity and the metabolic syndrome. Best Pract Res Clin Gastroenterol 2014;28(4):637-53.

[2] Farrell GC, Larter CZ. Nonalcoholic fatty liver disease: from steatosis to cirrhosis. Hepatol Baltim Md 2006;43(2 Suppl 1):S99-112.

[3] De Alwis NMW, Day CP. Non-alcoholic fatty liver disease: the mist gradually clears. J Hepatol 2008;48(Suppl 1):S104-12.

[4] Kotronen A, Yki-Järvinen H. Fatty liver: a novel component of the metabolic syndrome. Arterioscler Thromb Vasc Biol 2008;28(1):27-38.

[5] Brunt EM, Janney CG, Di Bisceglie AM, et al. Nonalcoholic steatohepatitis: a proposal for grading and staging the histological lesions. Am J Gastroenterol 1999;94(9):246774.

[6] Targher G, Arcaro G. Non-alcoholic fatty liver disease and increased risk of cardiovascular disease. Atherosclerosis 2007;191(2):235-40.

[7] Amarapurkar D, Kamani P, Patel N, et al. Prevalence of non-alcoholic fatty liver disease: population based study. Ann Hepatol 2007;6(3):161-3.

[8] Clark JM. The epidemiology of nonalcoholic fatty liver disease in adults. J Clin Gastroenterol 2006;40(Suppl 1):S5-10.

[9] Mohan V, Deepa R. Adipocytokines and the expanding Asian Indian phenotype. J Assoc Physicians India 2006;54:685-6.
[10] Mohan V, Deepa M, Deepa R, et al. Secular trends in the prevalence of diabetes and impaired glucose tolerance in urban South India--the Chennai Urban Rural Epidemiology Study (CURES-17). Diabetologia 2006;49(6):1175-8.

[11] Day CP. Pathogenesis of steatohepatitis. Best Pract Res 2002;16(5):663-78.

[12] Koo SH. Nonalcoholic fatty liver disease: molecular mechanisms for the hepatic steatosis. Clin Mol Hepatol 2013;19(3):210-15.

[13] Larter CZ, Yeh MM, Haigh WG, et al. Dietary modification dampens liver inflammation and fibrosis in obesityrelated fatty liver disease. Obesity (Silver Spring) 2013;21(6):1189-99.

[14] Xu R, Huang H, Zhang Z, et al. The role of neutrophils in the development of liver diseases. Cell Mol Immunol 2014;11(3):224-31.

[15] Alkhouri N, Morris-Stiff G, Campbell C, et al. Neutrophil to lymphocyte ratio: a new marker for predicting steatohepatitis and fibrosis in patients with nonalcoholic fatty liver disease. Liver Int 2012;32(2):297-302.

[16] Singh D, Das CJ, Baruah MP. Imaging of non alcoholic fatty liver disease: a road less travelled. Indian J Endocrinol Metab 2013;17(6):990-5.

[17] Yilmaz H, Yalcin KS, Namuslu $M$, et al. Neutrophillymphocyte ratio (NLR) could be better predictor than Creactive protein (CRP) for liver fibrosis in non-alcoholic steatohepatitis (NASH). Ann Clin Lab Sci 2015;45(3):27886.

[18] Bataller R, Brenner DA. Liver fibrosis. J Clin Invest 2005;115(2):209-18.

[19] Fujii H, Kawada N. Inflammation and fibrogenesis in steatohepatitis. J Gastroenterol 2012;47(3):215-25.

[20] Tamhane UU, Aneja S, Montgomery D, et al. Association between admission neutrophil to lymphocyte ratio and outcomes in patients with acute coronary syndrome. Am J Cardiol 2008;102(6):653-7.

[21] Bhutta H, Agha R, Wong J, et al. Neutrophil-lymphocyte ratio predicts medium-term survival following elective major vascular surgery: a cross-sectional study. Vasc Endovascular Surg 2011;45(3):227-31.

[22] Gomez D, Farid S, Malik HZ, et al. Preoperative neutrophilto-lymphocyte ratio as a prognostic predictor after curative resection for hepatocellular carcinoma. World J Surg 2008;32(8):1757-62.

[23] Bhatti I, Peacock 0, Lloyd G, et al. Preoperative hematologic markers as independent predictors of prognosis in resected pancreatic ductal adenocarcinoma: neutrophil - lymphocyte versus platelet-lymphocyte ratio. Am J Surg 2010;200(2):197-203.

[24] Yamanaka T, Matsumoto S, Teramukai S, et al. The baseline ratio of neutrophils to lymphocytes is associated with patient prognosis in advanced gastric cancer. Oncology 2007;73(3-4):215-20.

[25] Cho H, Hur HW, Kim SW, et al. Pre-treatment neutrophil to lymphocyte ratio is elevated in epithelial ovarian cancer and predicts survival after treatment. Cancer Immunol Immunother 2009;58(1):15-23.

[26] Basaranoglu M, Neuschwander-Tetri BA. nonalcoholic fatty liver disease: clinical features and pathogenesis. Gastroenterol Hepatol 2006;2(4):282-91. 
[27] Singh SP, Singh A, Misra D, et al. Risk factors associated with non-alcoholic fatty liver disease in Indians: a casecontrol study. J Clin Exp Hepatol 2015;5(4):295-302.

[28] Chalasani N, Younossi Z, Lavine JE, et al. The diagnosis and management of non-alcoholic fatty liver disease: practice guideline by the American Association for the Study of Liver Diseases, American College of Gastroenterology and the American Gastroenterological Association. Hepatology 2012;55(6):2005-23.

[29] Kumar R, Rastogi A, Sharma MK, et al. Clinicopathological characteristics and metabolic profiles of non-alcoholic fatty liver disease in Indian patients with normal body mass index: do they differ from obese or overweight nonalcoholic fatty liver disease? Indian J Endocrinol Metab 2013;17(4):665-71.

[30] Kuppan G, Anjana RM, Deepa M, et al. Inflammatory markers in relation to nonalcoholic fatty liver disease in urban South Indians. Diabetes Technol Ther 2012;14(2):152-8.
[31] Liu F, Zhou H, Ca L, et al. Risk of reduced platelet counts in nonalcoholic fatty liver disease (NAFLD): a prospective cohort study. Lipids Health Dis 2018;17(1):221.

[32] Chang Y, Ryu S, Sung E, et al. Higher concentrations of alanine aminotransferase within the reference interval predict nonalcoholic fatty liver disease. Clin Chem 2007;53(4):686-92.

[33] Byun JS, Yi HS. Hepatic immune microenvironment in alcoholic and nonalcoholic liver disease. Biomed Res Int 2017;2017:6862439.

[34] Firneisz G. Non-alcoholic fatty liver disease and type 2 diabetes mellitus: the liver disease of our age? World J Gastroenterol 2014;20(27):9072-89.

[35] Chang Y, Ryu S, Zhang Y, et al. A cohort study of serum bilirubin levels and incident non-alcoholic fatty liver disease in middle aged Korean workers. PLoS One 2012;7(5):e37241. 